AIAA-2001-4016

\title{
ESTIMATION OF AIRCRAFT UNSTEADY AERODYNAMIC PARAMETERS FROM DYNAMIC WIND TUNNEL TESTING
}

\author{
Patrick C. Murphy* \\ NASA Langley Research Center \\ Hampton, Virginia USA 23681-2199 \\ Vladislav Klein ${ }^{\dagger}$ \\ The George Washington University \\ NASA Langley Research Center \\ Hampton, Virginia USA 23681-2199
}

\begin{abstract}
Improved aerodynamic mathematical models, for use in aircraft simulation or flight control design, are required when representing nonlinear unsteady aerodynamics. A key limitation of conventional aerodynamic models is the inability to map frequency and amplitude dependent data into the equations of motion directly. In an effort to obtain a more general formulation of the aerodynamic model, researchers have been led to a parallel requirement for more general testing methods. Testing for a more comprehensive model can lead to a very time consuming number of tests especially if traditional single frequency harmonic testing is attempted. This paper presents an alternative to traditional singlefrequency forced-oscillation testing by utilizing Schroeder sweeps to efficiently obtain the frequency response of the unsteady aerodynamic model. Schroeder inputs provide signals with a flat power spectrum over a specified frequency band. For comparison, experimental results using the traditional single-frequency inputs are also considered. A method for data analysis to determine an adequate unsteady aerodynamic model is presented. Discussion of associated issues that arise during this type of analysis and comparison of results using traditional single frequency analysis are provided.

* Senior Research Engineer, Senior Member AIAA

$\uparrow$ Professor Emeritus, Associate Fellow AIAA

Copyright $\odot 2001$ by the American Institute of Aeronautics and Astronautics, Inc. No copyright is asserted in the United States under Title 17, U.S. Code. The U.S. Government has a royaltyfree license to exercise all rights under the copyright claimed herein for Governmental purposes. All other rights are reserved by the copyright owner.
\end{abstract}

\section{Nomenclature}

A, B, C

$a, \mathrm{~b}_{1}$

$\bar{c}$

$\mathrm{C}_{\mathrm{L}}$

$\mathrm{Cm}$

$\mathbf{J}$

$\mathrm{k}$

$l$

$\mathrm{N}$

PSD

q

s

$\mathrm{t}$

V

$\mathrm{V}$

Z

$\alpha$

$\theta$

$\sigma^{2}$

$\tau$

$\omega$

superscripts

estimate

subscripts

L lift force.

m pitching moment 


\section{Introduction}

Conventional aerodynamic math models for use in aircraft simulation or flight control design have become increasingly deficient as aircraft maneuvering capabilities have extended the flight envelope beyond conventional boundaries. In these new flight regimes, especially cases where aircraft maneuver at high angular rate or high angle of attack $(\alpha)$, nonlinear unsteady aerodynamic effects associated with rigid body responses have been well documented. The need to improve the aerodynamic mathematical model in these cases is generally acknowledged ${ }^{1-5}$. Although the aerodynamic modeling problem is readily identified with military fighter aircraft, it is also an issue for civilian transport aircraft. Airliner loss-of-control accidents due to adverse weather or system failures place these aircraft in nonlinear flight regimes that are not well modeled.

The deficiency in most aircraft simulations and models used in control design is the use of a traditional formulation for the aerodynamic model ${ }^{6}$. This formulation assumes that the aerodynamic forces and moments can be represented by a differentiable function and therefore expanded in a Taylor Series expansion with only first order terms (stability and control derivatives) retained. Although this mathematical structure is adequate for benign portions of the flight envelope, it is completely incapable of modeling aircraft responses when nonlinear unsteady effects occur. Information about the aerodynamics can be obtained from static and dynamic wind tunnel testing.

A key limitation of the conventional model structure is inability to map frequency and amplitude dependent dynamic data into the equations of motion directly. In addition, this formulation only accommodates the measurement of combined stability derivatives during wind tunnel oscillatory tests preventing separate estimates of damping and unsteady terms. Because the conventional combined terms are frequency, amplitude, and angle of attack dependent, simulation engineers are forced to find ad hoc methods to incorporate unsteady effects into the equations of motion $^{5}$.

In an effort to obtain a more general formulation of the aerodynamic model, researchers have been led to a parallel requirement for more general testing methods. Tobak ${ }^{7}$ and others have suggested testing methods that allow measurement of the basic parameters of a general aerodynamic model. Testing for a more comprehensive model can lead to a very large number of tests, especially if traditional single frequency harmonic testing is attempted. This paper offers an alternative to traditional single-frequency testing by utilizing Schroeder ${ }^{8}$ sweeps to obtain the unsteady aerodynamic model. Schroeder inputs provide signals with a flat power spectrum over a specified frequency band with low peak-to-peak input amplitudes. Amplitudes are controlled by properly phasing each harmonic.

The modeling of aircraft aerodynamics in planar one degree-of-freedom motion in pitch is identical to that in Refs. [12] and [13]. For data analysis the least squares and maximum likelihood estimates were used. The methodology is tested on wind tunnel data from a $10 \%$ scale model of an F-16XL configuration. The results are compared with those obtained from traditional static and single frequency data in Ref. [12] and large amplitude oscillatory data in Ref. [13].

\section{Modeling and Identification}

Results from wind tunnel forced-oscillation tests for given amplitude and mean angle of attack show that the resulting combination of stability derivatives depends on the frequency of the oscillations. This dependency contradicts the basic assumption that stability derivatives are time invariant. The effect of frequency on the aerodynamic parameters was explained by Goman, ${ }^{9} 10$ et al., at TsAGI, and by Klein ${ }^{11,12}$ et al., at NASA LaRC, by formulating the linear aerodynamic model equations with unsteady terms.

As an example, lift is considered in the form

$$
\begin{aligned}
C_{L}(t)=C_{L}(0) & +\int_{0}^{t} C_{L \alpha}(t-\tau) \dot{\alpha}(\tau) d \tau \\
& +\frac{l}{V} \int_{0}^{t} C_{L_{q}}(t-\tau) \dot{q}(\tau) d \tau
\end{aligned}
$$

where $C_{L_{\alpha}}(t)$ and $C_{L_{q}}(t)$ are the indicial functions, $C_{L}(0)$ is the initial value of $C_{L}, l$ is the characteristic length, and $\mathrm{V}$ is the airspeed. Two assumptions were adopted to allow simplification of the model used in the analysis of measured data: a) the effect of $\dot{q}(t)$ on the lift can be neglected and $b$ ) the indicial function $C_{L_{\alpha}}(t)$ can be expressed as 


$$
C_{L_{\alpha}}(t)=C_{L_{\alpha}}(\infty)-a e^{-b_{1} t}
$$

The simplified model, which takes into account increments with respect to steady state conditions, can be expressed as

$$
\begin{array}{r}
C_{L}(t)=C_{L_{\alpha}}(\infty) \alpha(t)+\frac{l}{V} C_{L_{q}}(\infty) q(t) \\
-a \int_{0}^{t} e^{-b_{1}(t-\tau)} \dot{\alpha}(\tau) d \tau
\end{array}
$$

where $C_{L_{\alpha}}(\infty)$ and $C_{L_{q}}(\infty)$ are the rates of change with $\alpha$ and q evaluated in steady flow.

Applying the Laplace transform to Eq. (3), the expression for the lift coefficient is obtained as

$$
C_{L}(s)=\frac{A s^{2}+B s+C}{s+b_{1}} \alpha(s)
$$

where $s$ is the Laplace transform parameter.

The problem addressed in this paper is the identification of the model given in (4). The parameters in (4) can be estimated by applying the maximum likelihood principle in frequency domain to the dynamic wind tunnel data. The model used in the estimation procedure has the form

$$
\begin{aligned}
& C_{L}(\omega)=\frac{-A \omega^{2}+C+i B \omega}{b_{1}+i \omega} \alpha(\omega) \\
& z(j)=C_{L}(j)+v(j), \quad j=1,2, \ldots, N
\end{aligned}
$$

where $C_{L}(w)$ and $\alpha(w)$ are the Fourier transforms of $C_{L}(t)$ and $\alpha(t), \mathrm{v}(j)$ is the measurement noise assumed to be a Gaussian random complex sequence with zero mean and variance $\sigma^{2}, \mathrm{~N}$ is the number of frequencies at which the transformed input output data are known, and $\omega$ is the angular frequency.

The maximum likelihood estimator minimizes the negative logarithm of the likelihood function

$$
\hat{\theta}=\min _{\theta, \sigma^{2}}\left\{-\ln L\left(Z_{N} ; \theta, \sigma^{2}\right)\right\}
$$

where $Z_{N}=[z(1), z(2), \ldots, z(N)]$ is a vector of output measurements and $\theta=\left[A, B, C, b_{1}\right]$ is the vector of unknown parameters. The parameters in (3) were obtained by solving the equations

$$
\begin{gathered}
A=\frac{\ell}{V} C_{L_{q}}(\infty) \\
B=C_{L_{\alpha}}(\infty)-a+b_{1} \frac{\ell}{V} C_{L_{q}}(\infty) \\
C=b_{1} C_{L_{\alpha}}(\infty) .
\end{gathered}
$$

Because Eq. (5) is nonlinear in the parameters, the estimation represents a nonlinear estimation problem. The initial values of parameters for this technique were obtained from a linear regression using the cost function

$$
J(\theta)=\sum_{j=1}^{N}\left|C_{L}(j)\left(b_{1}+i \omega_{j}\right)+\left(A \omega_{j}^{2}-C-i B \omega_{j}\right) \alpha(j)\right|^{2}
$$

\section{$\underline{\text { Experiment }}$}

A drawing of the $10 \%$ scale F-16XL model is shown in Figure 1. Dynamic tests were conducted in the NASA Langley 12-Foot Low-Speed Wind Tunnel. For these tests the model was mounted on a dynamic test rig through a six-component strain-gauge balance. The dynamic test rig is a computer controlled hydraulically actuated system, which was stingmounted on a C-strut support system. The mounting arrangement rotated the model about the reference center of gravity location of $0.558 \bar{c}$. The tests were conducted at a dynamic pressure of $192 \mathrm{~Pa}$ (4 psf) resulting in the flow velocity of $17.52 \mathrm{~m} / \mathrm{sec}(58 \mathrm{fps})$ and a Reynolds number of $10^{6}$ based on the mean aerodynamic chord.

Oscillatory data were created using Schroeder sweeps in $\alpha$ as an input at 13 mean values of angle of attack, $\alpha_{0}$, and an amplitude, $\alpha_{\mathrm{A}}=5$ degrees. The range of $\alpha$-mean values was from $\alpha_{0}=0$ to 65 degrees. Data were sampled at $100 \mathrm{~Hz}$ with an analog filter at $10 \mathrm{~Hz}$. Tests were repeated ten times at each angle of attack and then an average signal was formed using the ensemble data. The ensemble-averaged data was used for data analysis.

Example time histories of angle of attack, lift and pitching moment coefficients, $\alpha, \mathrm{C}_{\mathrm{L}}$, and $\mathrm{Cm}$, at $\alpha_{0}=$ 36 degrees is given in Figure 2. These plots show displacements relative to starting values at $\alpha_{0}$. The harmonic content of the angle of attack is shown in Figure 3 as a function of reduced frequency, k. Figure 3 indicates a flat spectrum for a frequency range of 0.03 $\mathrm{Hz}$ to $2.0 \mathrm{~Hz}$ which corresponds to a range of nondimensional frequencies $\mathrm{k}=0.004$ to 0.268 . For the 
analysis, time histories of $\alpha$ and the longitudinal aerodynamic coefficients were transformed to the frequency domain using a Discrete Fourier Transform (DFT) algorithm ${ }^{14}$. This algorithm allowed the transform to be performed over the frequency range of 0 to $2 \mathrm{~Hz}$.

In addition, the traditional in-phase and out-of phase components of aerodynamic coefficients were computed from transformed data. Expressing transformed angle of attack and lift coefficients as $\alpha(\omega)=u_{1}+i v_{2}$ and $C_{L}(\omega)=u_{2}+i v_{2}$, it can be shown that the transfer function

$$
\frac{C_{L}(\omega)}{\alpha(\omega)}=\frac{u_{2}+i v_{2}}{u_{1}+i v_{1}}=U+i V
$$

Then the in-phase and out-phase components are, respectively,

$$
\begin{aligned}
& \bar{C}_{L_{\alpha}}=C_{L_{\alpha}}-k^{2} C_{L \dot{q}}=U \text { and } \\
& \bar{C}_{L_{q}}=C_{L_{q}}+C_{L \dot{\alpha}}=\frac{1}{k} \mathrm{~V} .
\end{aligned}
$$

Both Fourier components are obtained from measured data and plotted against angle of attack for three values of reduced frequency. These plots are presented in Figure 4.

\section{Results and Discussion}

The estimation method used in this study is different from that used in References [11-13] because it provides estimates of all unknown parameters at different values of $\alpha$. The previous methods in References [11] and [12] assumed that the time constant, $1 / \mathrm{b}_{1}$, did not change with $\alpha$. In Reference [13] it was assumed that initial estimates of $C_{L_{q}}(\infty)$ were available from small-amplitude oscillation data to initialize estimation of the unsteady term in Eq. (3).

The parameter estimation method outlined in this paper was applied to longitudinal (planar) lowamplitude forced-oscillation data. The method was only applied to cases in the range of angle of attack between $\alpha=20$ degrees and $\alpha=65$ degrees. This range of angle of attack is where unsteady effects primarily occur for this model. The estimated parameters in Eq. (3) and their 2-sigma bounds are shown in Figures 5 and 6 for the lift and pitching moment equations, respectively. The figures also show alternate estimates of the parameters when possible.
Figure 5 presents estimates of unknown parameters in model Eq. (3). The estimation process failed for $\alpha<25$ degrees because of the lack of unsteady information in the measurement data. This identifiability issue is corroborated in Figure 4, where the variation of the in-phase and out-of-phase components with frequency is greatly reduced. Below $\alpha$ $=25$ degrees there is virtually no variation with frequency.

Estimates of $C_{L_{\alpha}}(\infty)$ show very good agreement with results obtained from static measurements. Static data estimates were obtained by performing a simple finite difference calculation on $\mathrm{C}_{\mathrm{L}}(\alpha)$. Estimates of $C_{L_{q}}(\infty)$, on the other hand, did not show good agreement with values in Ref. [12] and error bounds were relatively large. This result is not too surprising since $C_{L_{q}}(\infty)$ is usually a very small contributor to lift and therefore difficult to estimate. Estimates of parameter a, the gain on the unsteady term, agreed very well with two other independent techniques in References [12] and [13]. Again the error bounds are large where the information content of the measured data is substantially reduced. Estimates of $b_{1}$ generally follow the same variation with $\alpha$ as that found in Reference [13]. The match with Reference [12] is only approximate since the parameter was assumed to be constant in that study to minimize identifiability issues.

In Figure 6, for $\alpha<45$ degrees, estimates of $C_{m_{\alpha}}(\infty)$ show very good agreement with estimates obtained from simple finite difference calculations on the static data. Increasing error bounds for estimates in the high and low $\alpha$ ranges occur as before due to low information content. Poor signal-to-noise ratio in the pitching moment channel also contributed to poor identifiability, particularly in the high $\alpha$ range. In Figure 2 an apparently more noisy response is seen in the pitching moment channel at $\alpha=36$ degrees. Signalto-noise ratio deteriorated further with increasing angle of attack. This problem was aggravated by the limited capability of the dynamic rig to follow low-amplitude oscillation commands. The rig was originally intended for large amplitude and relatively fast oscillations. In this low-amplitude experiment the Schroeder sweep required a number of small oscillations as small as one degree, particularly at the beginning of the sweep (see Figure 2). The pitch channel was particularly sensitive to this problem and for the $\alpha=46$ degrees case convergence for the estimation algorithm was poor. Further investigation is required to fully explain the 
pitching moment response. Excluding the $\alpha=63$ degrees case, estimates of $C_{m_{q}}(\infty)$ match very well with values given in Ref. [13] and the 2-sigma bounds were relatively small. Estimates of parameter a, for the pitching moment equation, also agreed very well with the independent technique in Ref. [13] for $\alpha<45$ degrees. For $\alpha>45$ degrees, estimates differed from Ref. [13] and large error bounds were obtained that reflect the poor information content and low signal-tonoise ratio. In addition, parameter a tended to be very small in the low and high $\alpha$ ranges making the estimation more difficult. Estimates of $b_{1}$ show a trend toward zero or small values for $\alpha$ above 45 degrees, thus indicating a trend toward neutral stability. With parameter a reduced the unsteady effect in $C_{m}$ is limited for $\alpha$ greater than 45 degrees. As mentioned previously, the estimates of $b_{1}$ from Ref. [12] assumed no dependence on $\alpha$. Estimates of the same parameters in Ref. [13] didn't reveal any dependencies on $\alpha$. Below 45 degrees, estimates of $b_{1}$ were in the same range as estimates from both Refs. [12] and [13].

Figures 7 and 8 show the frequency response of the lift and pitching moment coefficients, respectively, during forced oscillations at $\alpha=36$ degrees. These figures show the relatively large measurement noise (frequency domain) for this study. Amplitude ratio and phase plots for the lift coefficient are relatively flat at the high frequency range $(2 \mathrm{~Hz})$. The pitching moment amplitude ratio is small and slightly increasing with frequency.

An identifiability issue of concern for the transfer function model structure given in Eq. (10) is pole/zero cancellation. Figure 9 shows the pole and two zeros as a function of $\alpha$ for the lift transfer function. The $2^{\text {nd }}$ zero shown as a diamond in the upper plot is relatively large and has a wide frequency separation from the other transfer function roots in the lower angle of attack range. The pole and $1^{\text {st }}$ zero are fairly close in magnitude and become almost equal at very low angle of attack. The lower plot highlights the pole and $1^{\text {st }}$ zero to show how the two approach cancellation at $\alpha=$ 20 degrees. At that point the values are within 5\% of each other creating a more difficult estimation problem. As the pole and $1^{\text {st }}$ zero values approach equality the model structure in Eq. (10) becomes inadequate and the estimation algorithm fails.

\section{Concluding Remarks}

This study is part of an ongoing effort at NASA Langley to develop a more general formulation of the aerodynamic model for aircraft that includes nonlinear unsteady aerodynamics. The general structure proposed for the aerodynamic model has the form

$$
\begin{aligned}
& L=L_{\text {static }}+L_{\text {dynamic }}+L_{\text {unsteady }} \\
& L_{\text {unsteady }}=\frac{1}{2} \rho V^{2} S C_{L}^{*}(t)
\end{aligned}
$$

where

$$
\dot{C}_{L}^{*}=-b(\alpha) C_{L}^{*}+a(\alpha) \dot{\alpha}
$$

Similar equations can be written for drag and pitching moment equations. This structure allows easy interpretation of the model parameters by retaining conventional stability and control derivatives for static and dynamic terms. Unsteady terms are obtained by solving a first order differential equation with $\alpha$ dependent coefficients. This approach offers a straightforward model for estimation and simulation.

Modeling unsteady aerodynamics demands substantial testing in wind tunnels and in turn a parallel requirement for more general and efficient test methods. In support of these goals this study demonstrated an alternative to single-frequency harmonic testing using Schroeder sweeps. A transfer function model that includes unsteady aerodynamics was developed. A frequency domain method for data analysis that can be used to estimate the unsteady aircraft models was presented. Identifiability issues associated with this methodology and with this particular experiment were discussed.

The methodology in this study compared well with previous studies that used different techniques to obtain the aerodynamic model for the F-16XL. Both present and past techniques separated the static, rotary, and unsteady terms and both modeled the unsteady term as an indicial function. However, the previous techniques made different assumptions about $a$ priori information. The previous methods assumed that some of the parameters were known. The present approach has the advantage that all unknown parameters in the model are estimated at once for various angle of attack.

The previous techniques only used conventional single-harmonic, constant-amplitude inputs requiring a substantially larger number of tests. This study demonstrated the use of Schroeder sweeps that provided wide-band, flat-spectra inputs with low peakto-peak input amplitudes. The maximum amplitude for the Schroeder sweep is constrained by the dynamic rig's capabilities and the bandwidth required for the 
experiment. The Schroeder algorithm prescribes smaller amplitude cycles within the sweep that improve the input from an experiment design point of view, especially for nonlinear or input-amplitude dependent systems. In general, the Schroeder sweep is an excellent frequency-rich input for forced oscillation testing however the dynamic rig must be able to handle the variety of input amplitudes and frequencies. When test rig capabilities are limited, an alternate choice may be a traditional frequency sweep with limited amplitude variation.

Identifiability issues for the unsteady model, discussed in this paper, were associated with either a lack of information content or an inadequate model structure. Degradation of information content in system response was found in three cases. First, low levels of frequency dependence were found for in-phase and outof-phase coefficients in the low and high $\alpha$ ranges. Second, low signal-to-noise ratios were found in pitching moment responses, especially in the high $\alpha$ range or low amplitude cases. Third, when parameter values were very small causing little contribution to the overall response. Inadequate model structure was highlighted in this study primarily for the problem of pole-zero cancellation at very low angles of attack. Other contributors to a mismatch between model structure and system response are suspected, i.e., the dynamic rig, designed for large amplitude motion, may have introduced responses not associated with the aerodynamics. This was especially a problem in the pitching moment channel at mid to high angles of attack and for small amplitude motion. In this study responses in the pitch channel did not completely follow the expected harmonic responses. Only minor distortion was expected since even at 5 degrees amplitude some nonlinear behavior can occur in the pitch channel.

\section{Acknowledgments}

Wind tunnel tests were carried out with the assistance of NASA researchers Jay Brandon, Dr. Sungwan Kim, and George Washington University student, Leslie Gould.

\section{References}

1. Brandon, J.M., and Foster, J.V.: Recent Dynamic Measurements and Considerations for Aerodynamic Modeling of Fighter Airplane Configurations, AIAA 98-4447, August 1998
2. Klein, V., and Noderer, K.D.: Modeling of Aircraft Unsteady Aerodynamic Characteristics", Part I - Postulated Models, NASA TM 109120, May, 1994; Part 2 Parameters Estimated From Wind Tunnel Data, NASA TM 110161, April 1995; Part 3 Parameters Estimated From Flight Data, NASA TM 110259, May 1996.

3. Greenwell, D.J.: Difficulties in the Application of Stability Derivatives to the Maneuvering Aerodynamics of Combat Aircraft, ICAS 983.9.2, September 1998, Melbourne, Australia.

4. Goman, M.G. (TsAGI), and Khrabrov, A.N.(TsAGI), State-Space Representation of Aerodynamic Characteristics of an Aircraft at High Angles of Attack, Journal of Aircraft, Vol.31, No.5, September-October, 1994, pp. 1109-1115.

5. Ogburn, M.E., Nguyen, L.T., and Hoffler, K.D.: Modeling of Large-Amplitude High Angle-ofAttack Maneuvers, AIAA 88-4357, Atmospheric Flight Mechanics Conference, August 1988.

6. Etkin, B.: Dynamics of Atmospheric Flight, John Wiley \& Sons, Inc., New York, 1972.

7. Tobak, Murray and Schiff, Lewis B.: On the Formulation of the Aerodynamic Characteristics in Aircraft Dynamics. NASA TR-R-456, 1976.

8. Schroeder, M.R.: Synthesis of Low-Peak-Factor Signals and Binary Sequences with Low Autocorrelation, IEEE Transactions on Information Theory, January 1970, pp. 85-89.

9. Goman, M. G.; Stolyarov, G. I.; Tartyshnikov, S. L.; Usolcev, S. P. and Khrabrov, A. N.: Mathematical Description of Longitudinal Aerodynamic Characteristics at High Angles of Attack Accounting for Dynamic Effects of Separated Flow. TsAGI Preprint No. 9, 1990 (in Russian).

10. Goman, M. and Khrabrov, A.: State-Space Representation of Aerodynamic Characteristics at High Angles of Attack. Journal of Aircraft, Vol. 31, No. 5, Sept.-Oct. 1994, pp. 1109-1115.

11. Klein, Vladislav and Noderer, Keith D.: Modeling of Aircraft Unsteady Aerodynamic Characteristics. Part 2-Parameters Estimated 
from Wind Tunnel Data. NASA TM 110161, 1995.

12. Klein, Vladislav; Murphy, Patrick C.; Curry, Timothy J. and Brandon, Jay M.: Analysis of Wind Tunnel Longitudinal Static and Oscillatory Data of the F-16XL Aircraft. NASA/TM-97206276, 1997.

13. Klein, V. and Murphy, P.C.: Estimation of Aircraft Nonlinear Unsteady Parameters from Wind Tunnel Data, NASA/TM-1998-208969, December 1998.

14. Morelli, Eugene A.: High Accuracy Evaluation of the Finite Fourier Transform Using Sampled Data, NASA TM 110340, June 1997.

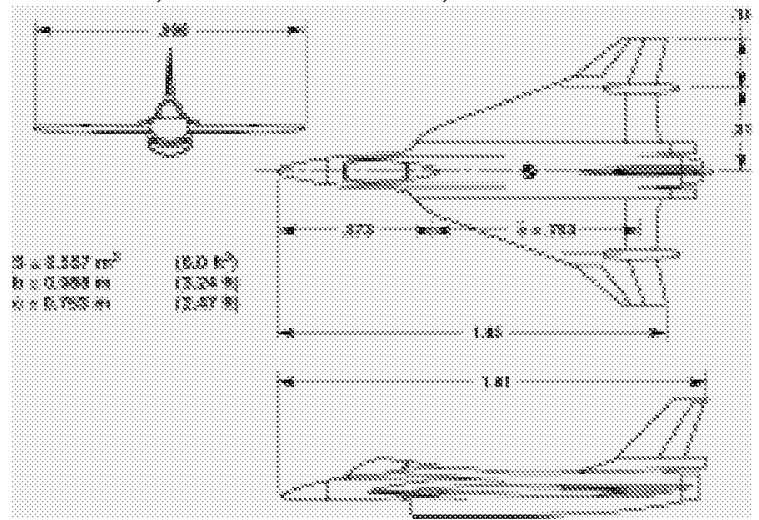

Figure 1. Three-view drawing of 10\% F-16XL model.
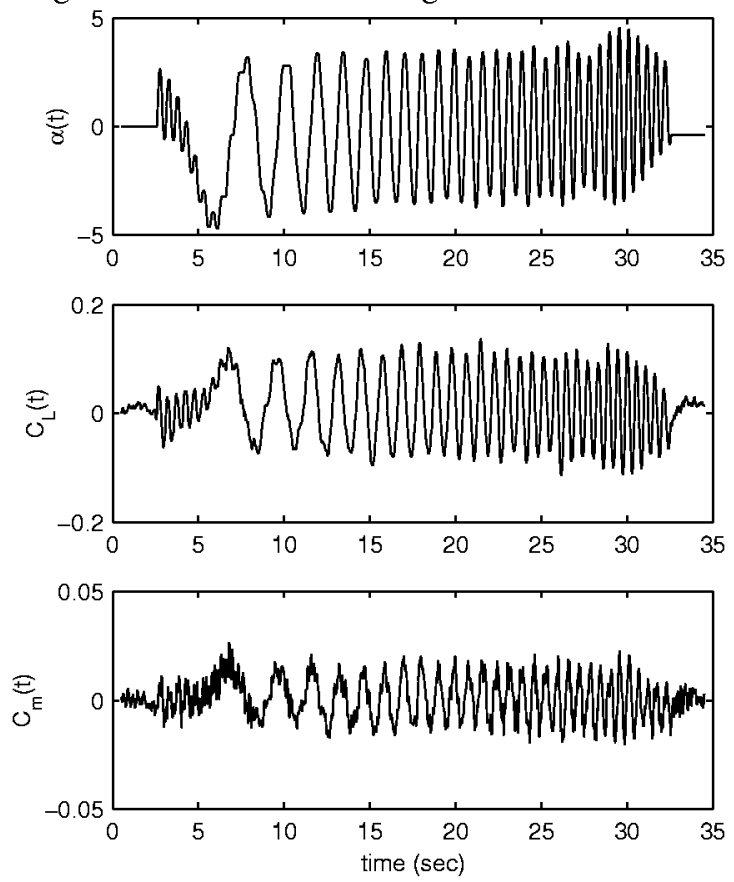

Figure 2. Time histories of angle of attack, lift, and pitch-moment coefficients for $\alpha_{0}=36$ degrees.

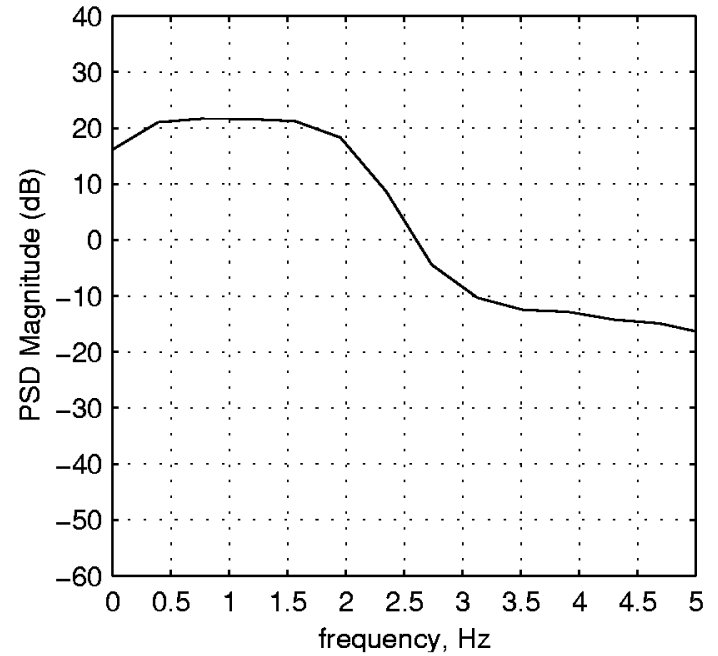

Figure 3. Harmonic content of transformed $\alpha$ input for $\alpha_{0}=36$ degrees.
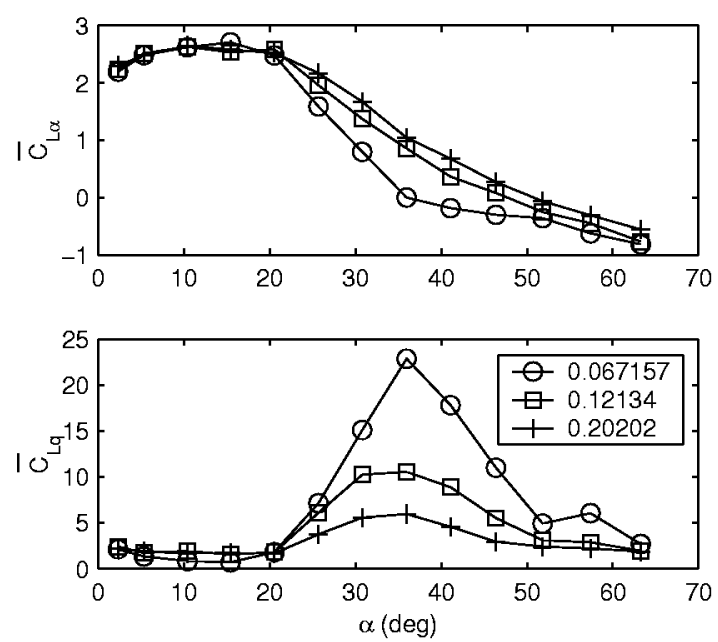

Figure 4. Variation of in-phase and out-of-phase components of lift coefficient with angle of atack for three different reduced frequencies. 

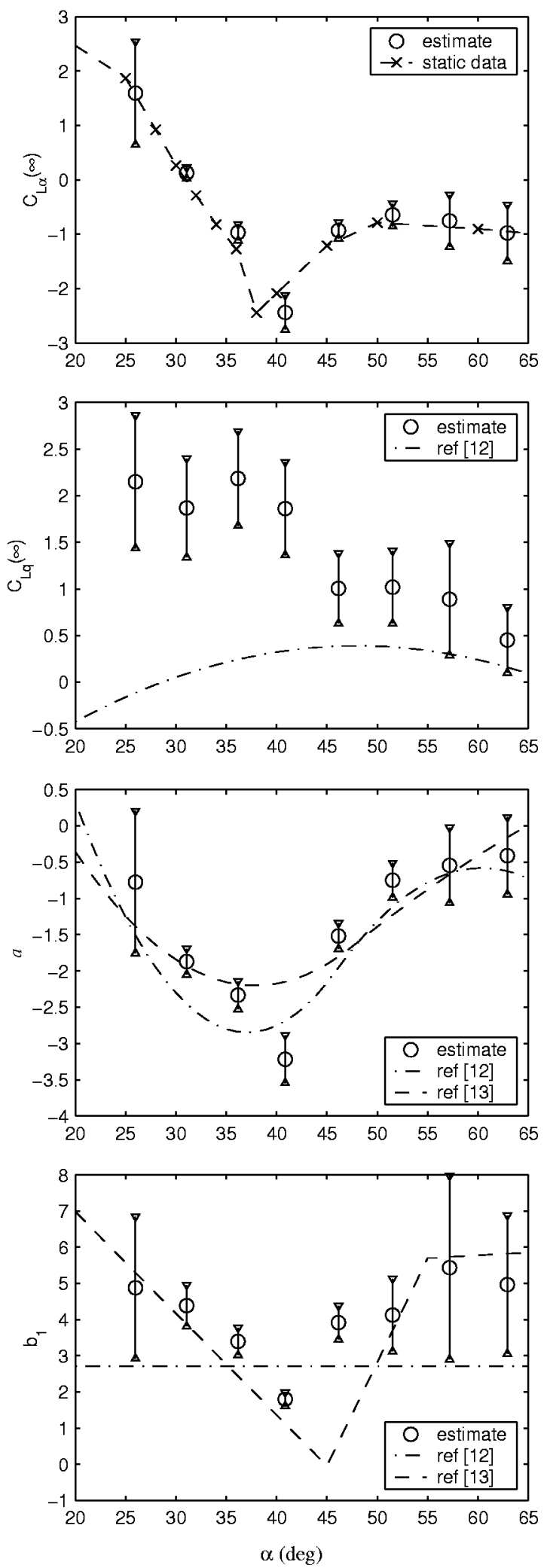

Figure 5. Estimated parameters and their $2 \sigma$ confidence bounds for lift coefficient.
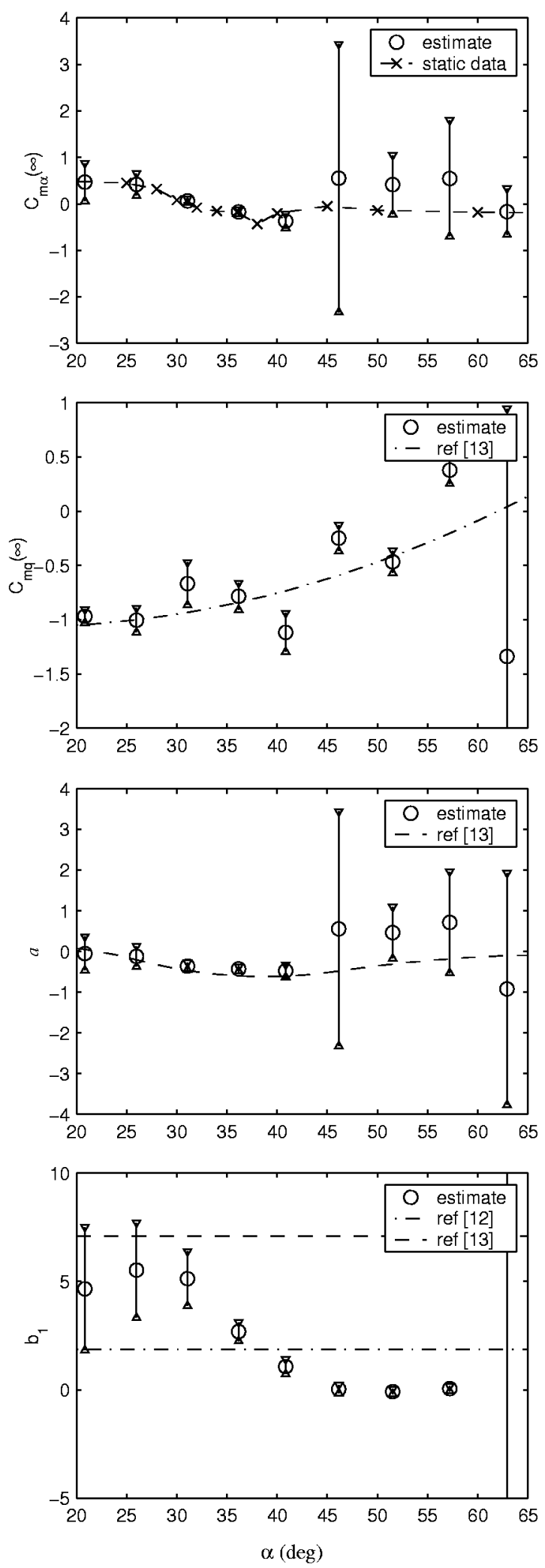

Figure 6. Estimated parameters and their $2 \sigma$ confidence bounds for pitch-moment coefficient. 

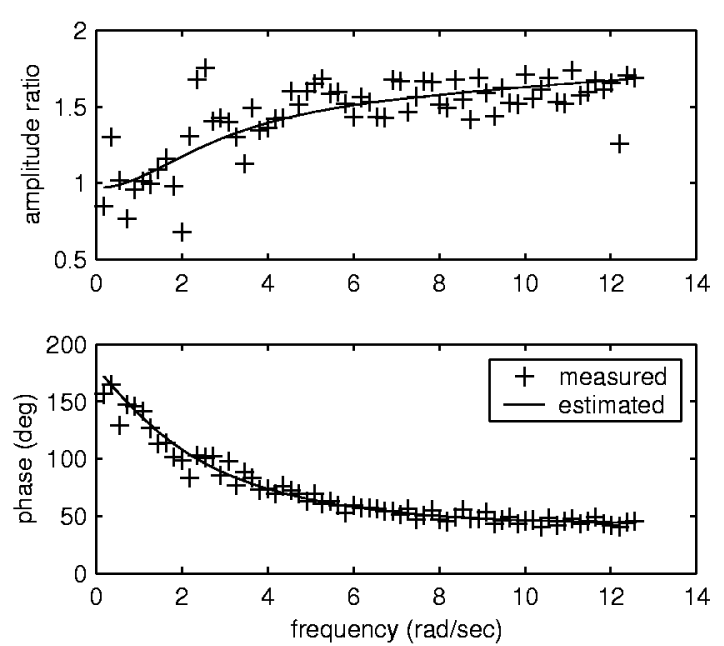

Figure 7. Comparison of measured and computed frequency response, $\mathrm{C}_{\mathrm{L}} / \alpha$.
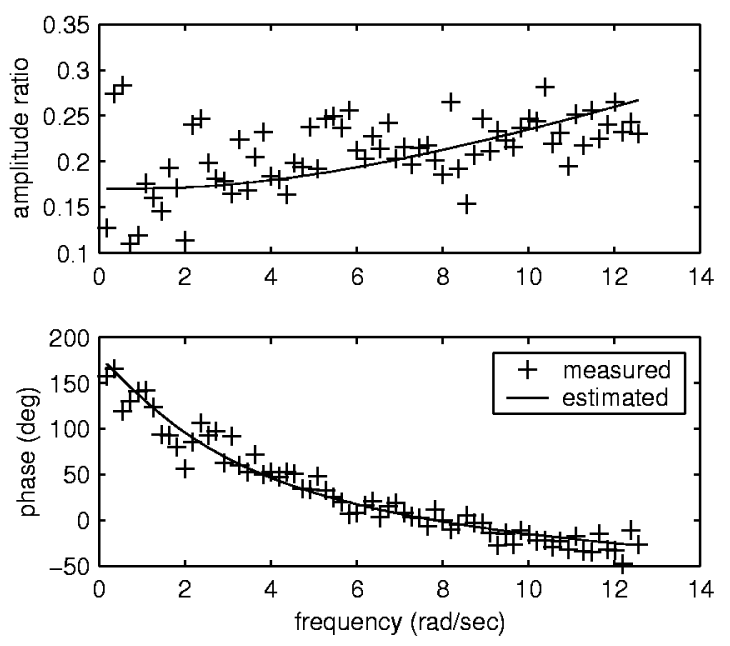

Figure 8. Comparison of measured and computed frequency response, $\mathrm{C}_{\mathrm{m}} / \alpha$.
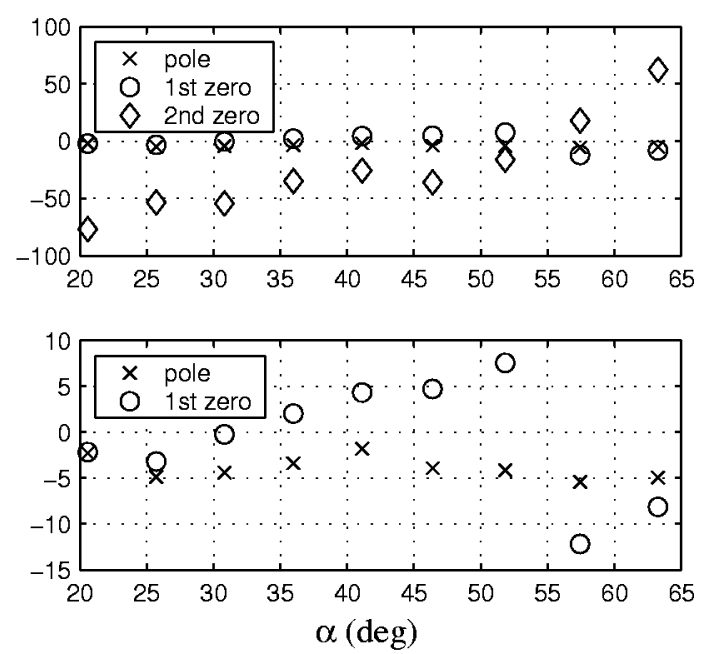

Figure 9. Pole and zero variation with angle of attack for lift coefficient transfer function. 